\title{
Selumetinib benefits children with inoperable plexiform neurofibromas
}

\begin{abstract}
4
...we hope that

our research

will facilitate

access of these

children to this

medication

that can both

shrink their

tumours and

make them

feel better...
\end{abstract}

Neurofibromatosis type 1 (NF1)

affects $\sim 1$ in 3,000 individuals

worldwide and is characterized by benign nerve sheath tumours, termed neurofibromas, that arise in infancy. Plexiform neurofibromas, which involve multiple branches of larger nerves, are commonly associated with pain, disfigurement, deficits in motor and neurological function, and a risk of malignant transformation. The mainstay treatments are surgery and pain management - no approved therapies exist for inoperable plexiform neurofibromas. Now, data from a phase II trial indicate that the MEK inhibitor selumetinib provides meaningful clinical benefit.

"This project was started owing to the unmet need of these patients with large debilitating tumours combined with the realization that some therapies developed for other cancers might also help these patients," explains Andrea Gross of the US National Cancer Institute (NCI). "Selumetinib blocks a molecular pathway that is known to be overactive in patients with NF1," she adds. Specifically, NF1 is caused by germline mutations in the gene encoding neurofibromin, which is a negative regulator of proliferative and survival signalling cascades

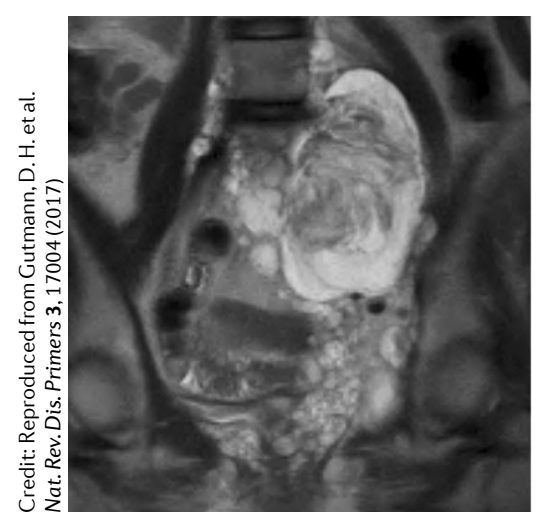

that include the RAS-MEK-ERK pathway. In a phase I NCI trial of this agent, durable partial responses (PRs) were observed in 17 (71\%) of 24 children with NF1-related plexiform neurofibromas, with anecdotal evidence of clinical benefits. "Given that these are not malignant tumours, we felt that, in order to truly demonstrate clinical benefit, we had to confirm not only tumour shrinkage but also improvements in symptoms and/or function; thus, we included extensive patient-reported and functional outcome measures as key secondary objectives in our phase II trial, in order to prospectively and systematically assess function, pain, disfigurement and quality of life (QOL)," Gross explains.

In this trial, 50 children with NF1 and symptomatic inoperable plexiform neurofibromas, including 21 (42\%) with progressive tumours at enrolment, received selumetinib twice daily. The patients had a median of three neurofibromarelated symptoms, most commonly disfigurement (in 88\%), motor dysfunction (66\%) and pain (52\%). In line with the phase I data, confirmed PRs occurred in 35 patients (70\%), lasting $\geq 1$ year in 28 (56\%). The median progression-free survival (PFS) duration was not reached and the 3-year PFS was $84 \%$ (compared with 1.3 years and $15 \%$, respectively, in a separate age-matched control cohort of 93 patients from a NCI naturalhistory study of NF1). Notably, responses were independent of age, tumour volume and progression status at baseline, and the location of the target lesion.

Adverse effects of treatment were mostly of grade $1-2$. However, nausea, vomiting, abdominal pain, diarrhoea, oral mucositis, rash, dry skin and/or elevated creatine phosphokinase levels each occurred in $>50 \%$ of patients. Toxicities resulted in dose reductions and treatment discontinuation in $28 \%$ and $10 \%$ of patients, respectively.

Importantly, the majority of patients had clinically meaningful improvements in self-reported tumour pain-intensity and interference of pain with daily functioning, in child-reported and/or parent-reported mobility and overall health-related QOL (including in physical, emotional and social domains), as well as in strength and range of motion. With regard to the global impression of change, some level of improvement in tumour-related problems was reported by $72 \%$ of children and $86 \%$ of parents. "By contrast, the natural-history study showed that, left untreated, these tumours generally grow and tumour-related complications tend to stay the same or get worse over time (not improve)," states Gross.

"Based primarily on the results of this trial, the maker of selumetinib has submitted a New Drug Application to the FDA for the treatment of NF1; we hope that our research will facilitate access of these children to this medication that can both shrink their tumours and make them feel better," says Gross. "However, there is definitely more work to be done looking for other potentially more effective drugs or drug combinations. We are also interested in possibly treating young children, who we know from the natural-history study have the most rapidly growing tumours, earlier in their lives to prevent them from ever developing serious and potentially life-threatening complications," she concludes.

David Killock

ORIGINAL ARTICLE Gross, A. M. et al. Selumetinib in children with inoperable plexiform neurofibromas. N. Engl.J. Med. https://doi.org/ 10.1056/NEJMoa1912735 (2020) 